\title{
A Case Study of Eukaryogenesis: The Evolution of Photoreception by Photolyase/Cryptochrome Proteins
}

\author{
Jennifer A. Miles ${ }^{1,2}$ (1) $\cdot$ Thomas A. Davies ${ }^{1} \cdot$ Robert D. Hayman $^{1} \cdot$ Georgia Lorenzen $^{1} \cdot$ Jamie Taylor $^{1}$. \\ Mubeena Anjarwalla ${ }^{1}$ - Sammie J. R. Allen ${ }^{1} \cdot$ John W. D. Graham ${ }^{1} \cdot$ Paul C. Taylor ${ }^{1,2}$
}

Received: 16 January 2020 / Accepted: 5 September 2020 / Published online: 26 September 2020

(c) The Author(s) 2020

\begin{abstract}
Eukaryogenesis, the origin of the eukaryotes, is still poorly understood. Herein, we show how a detailed all-kingdom phylogenetic analysis overlaid with a map of key biochemical features can provide valuable clues. The photolyase/cryptochrome family of proteins are well known to repair DNA in response to potentially harmful effects of sunlight and to entrain circadian rhythms. Phylogenetic analysis of photolyase/cryptochrome protein sequences from a wide range of prokaryotes and eukaryotes points to a number of horizontal gene transfer events between ancestral bacteria and ancestral eukaryotes. Previous experimental research has characterised patterns of tryptophan residues in these proteins that are important for photoreception, specifically a tryptophan dyad, a canonical tryptophan triad, an alternative tryptophan triad, a tryptophan tetrad and an alternative tetrad. Our results suggest that the spread of the different triad and tetrad motifs across the kingdoms of life accompanied the putative horizontal gene transfers and is consistent with multiple bacterial contributions to eukaryogenesis.
\end{abstract}

Keywords Cryptochrome $\cdot$ Photolyase $\cdot$ Cyanobacteria $\cdot$ Horizontal gene transfer $\cdot$ Eukaryogenesis

\section{Introduction}

It is widely accepted that eukaryogenesis involved an endosymbiosis between at least one archaeon, probably from the Asgard lineage, and at least one bacterium, most likely an ancestral $\alpha$-proteobacterium (López-García and Moreira 2019). More controversial and less well understood is the possibility of further symbioses or other significant gene flows from bacteria to ancestral eukaryotes (Pittis and Gabaldón 2016; Spang et al. 2019). Both the order in which these key evolutionary events occurred and their timeline are unclear. Characterising the emergence of animals from

Handling Editor: Alan Christensen.

Electronic supplementary material The online version of this article (https://doi.org/10.1007/s00239-020-09965-x) contains supplementary material, which is available to authorized users.

Paul C. Taylor

p.c.taylor@leeds.ac.uk

1 School of Chemistry, University of Leeds, Woodhouse Lane, Leeds LS2 9JT, UK

2 Astbury Centre for Structural Molecular Biology, University of Leeds, Woodhouse Lane, Leeds LS2 9JT, UK this ancient mix is of particular interest and offers a useful perspective from which to explore the major transitions (Paps 2018).

In addition to the large-scale phylogenomic studies that underpin our emerging understanding of eukaryogenesis, the field may benefit from case studies of the evolution of individual genes/proteins across the kingdoms of life. Researchers are finding surprising similarities in chemical signalling-related proteins between animals and groups of complex bacteria, including cyanobacteria (Brash et al. 2014; Magnani et al. 2017; Miles et al. 2019; Millard et al. 2014; Picciano and Crane 2019; Ponting et al. 1999; Rawlings 2015) that pose some important questions about horizontal gene transfer (HGT) in the origin of animals. This offers an alternative perspective from which to view the major transitions in eukaryogenesis. A good object of study would therefore be a family of proteins found in many major kingdoms and taxa, including animals, and that possesses a highly distinctive biochemical motif or motifs that would allow us to further refine phylogenetic analyses.

The photolyase/cryptochrome superfamily is distinguished biochemically by the ability of these proteins to harbour radical-pair intermediates (Hore and Mouritsen 2016). This phenomenon allows photolyases and cryptochromes to 
sense and react to sunlight, which is of enormous benefit to many species. There are two broad types of response, namely DNA repair and signalling (Mei and Dvornyk 2015). In particular, ultraviolet B (UVB) present in sunlight can be damaging to genetic material, changing the chemical structure of DNA, leading to aberrations such as cyclic pyrimidine dimers (CPDs) and (6-4) dimers. Once activated by light, photolyases are able to catalyse the reverse of some of these damaging reactions, restoring the original DNA structure (Sancar 2016). The chemical process is initiated by reduction of fully oxidised FAD to FAD $\cdot^{-}$or FADH. and finally through to $\mathrm{FADH}^{-}$, with the reduced species providing electrons to cleave bonds in the defective DNA (Müller et al. 2015). Alongside this is an antenna chromophore with a different absorption range that broadens spectral properties. Five of these antenna chromophores have been identified so far for photolyase/cryptochrome superfamily members (Kiontke et al. 2014). Alternatively, but based on the same chemistry, cryptochromes are core components of circadian rhythms, which allow organisms, including humans, to respond behaviourally to the daily cycle of light and dark, by initiating and shutting down biochemical processes as appropriate (Mei and Dvornyk 2015). Cryptochromes are also proposed to have a role in magnetoreception (Hore and Mouritsen 2016). Importantly for our work, the efficiency of the radical-pair mechanism appears to depend on precise details of the amino acid sequence of the cryptochrome in a given species, in particular a "dyad", "triad" or "tetrad" of tryptophan residues (Müller et al. 2015; Cailliez et al. 2016). We aimed to carry out a wide-ranging phylogenetic analysis of the photolyase/cryptochrome superfamily, building on earlier work (see below), and to map the presence of tryptophan motifs across the resulting phylogenetic tree.

The aim of this study was to ascertain the bacterial contribution to the function and evolution of the photolyase/ cryptochrome superfamily and hence inform models of eukaryogenesis. Previous phylogenetic analyses have taken place within this family (Kanai et al. 1997; Öztürk et al. 2007; Mei and Dvornyk 2015; Scheerer et al. 2015). The most recent comprehensive phylogenetic analysis of the photolyase/cryptochrome superfamily in eukaryotes was published by Mei and Dvornyk in 2015 (Mei and Dvornyk 2015), who noted the existence of prokaryotic orthologues but included only a few bacterial sequences in their analysis. Earlier, Lucas-Lledó and Lynch had undertaken another major phylogenetic analysis including numerous prokaryotic photolyase/cryptochromes (Lucas-Lledó and Lynch 2009). However, their study focused mainly on photolyase gene loss, whereas we are interested in the acquisition of particular biochemical features. Also, very many prokaryotic genomes have been sequenced since 2009. Therefore, we decided to start from the eukaryotic analysis from Mei and Dvornyk, to carry out our own search for prokaryotic orthologues and to cross-reference our findings to the work of Lucas-Lledó and Lynch. Previously it had been suggested that both gene duplication events, gene loss and convergent evolution may have taken place across this family of proteins, in particular during the evolution of cryptochromes from plants and animals, known as Plant Cry and Animal Cry, respectively (Cashmore et al. 1999).

\section{Results}

\section{Phylogenetic Analysis of Currently Available Prokaryotic and Eukaryotic Genomes Supports the Existence of Seven Subfamilies of Photolyase/ Cryptochromes}

To start our search for candidate bacterial orthologues, we selected seven of the sequences used by Mei and Dvornyk (2015) as queries for their analysis. Listed in the Materials and Methods, these cover the previously identified subfamilies (6-4) Photolyase/Animal Cry, Plant Cry, Plant Photolyase, Cyclobutane Pyrimidine Dimer (CPD) Class I Photolyase, CPD Class II Photolyase and Cry-DASH (Fig. 1). The top 20 candidate orthologues from different bacterial species were selected from protein-protein BLAST (BLASTp) searches of the NCBI non-redundant protein database, filtered for taxid:2 (bacteria). We found no candidate bacterial orthologues of Plant Photolyase proteins using this method. In order to confirm that the identified sequences were candidate orthologues, these were then used in a reciprocal BLASTp search against the original species to see if the starting sequence was obtained as the best match. Sequences with complete genomes were kept and sequences obtained from metagenomes were removed when the genome completion was below 50\%. Extra eukaryotic and archaeal sequences, beyond those used as start points for our BLASTp searches, were added to give a more complete picture of the photolyase/cryptochrome superfamily including species from most major taxa. We accrued 186 amino acid sequences in our database (full details in Supplementary Information).

According to eggNOG, $84 \%$ of orthologues of "cryptochrome/DNA photolyase, FAD-binding domain" are found in bacteria, $14 \%$ in eukaryota and $2 \%$ in archaea. The bacterial total of $84 \%$ comprises $45 \%$ from proteobacteria $(15 \%$ $\alpha$-, $6 \% \beta$ - and $22 \% \gamma$-proteobacteria), $16 \%$ from actinobacteria, $8 \%$ from bacteroidetes/chlorobi, $5 \%$ from cyanobacteria, $5 \%$ from firmicutes and 5\% other. A similar spread across the bacterial phyla is observed in OrthoDB. We have ensured there are representatives of all these phyla in our database.

We aligned our protein sequences using MUSCLE (Multiple Sequence Comparison by Log-Expectation) (Edgar 2004) to directly compare it to the analysis undertaken 
Fig. 1 The seven subfamilies used in phylogenetic analysis. Main representatives are shown alongside their predicted domains based on NCBI. PHR photolyase, FAD FAD-binding domain, CryC Cryptochrome $\mathrm{C}$

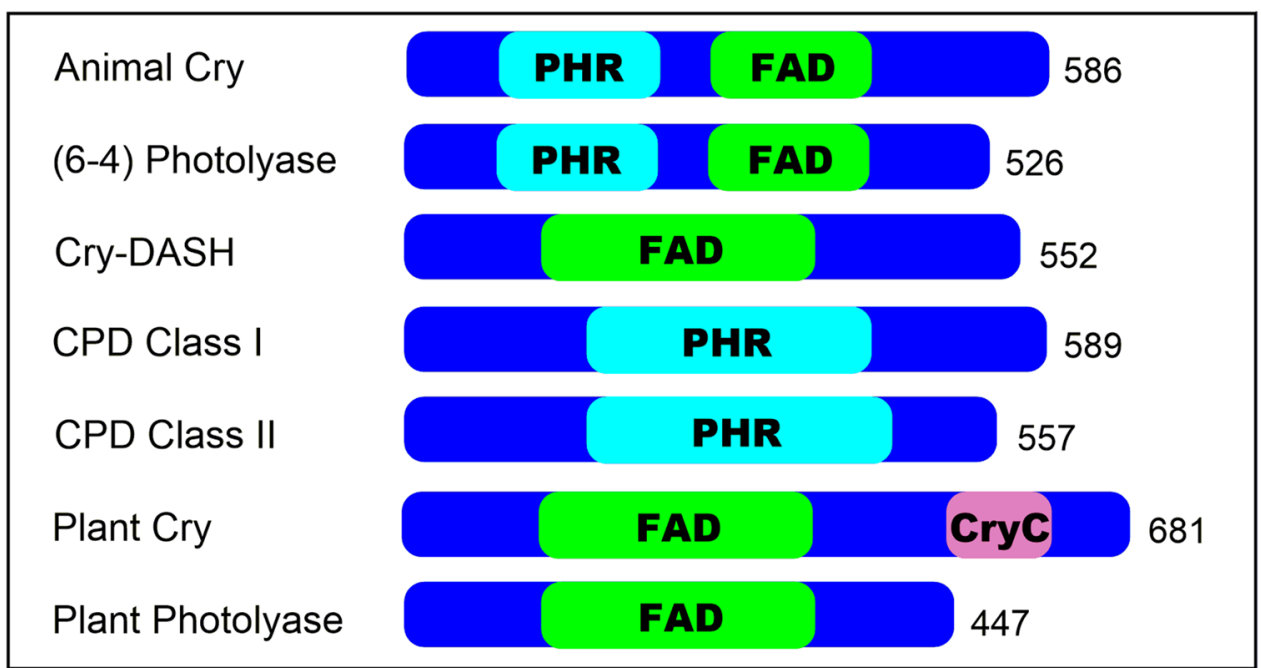

by Mei and Dvornyk. To reinforce the reliability we also aligned our chosen sequences using MAFFT (Multiple Alignment using Fast Fourier Transform) (Katoh et al. 2017) and PRANK (Löytynoja and Goldman 2010) for further comparison. Following alignment and trimming of sequences using Gblocks (Castresana 2000), trees were generated using PhyML with approximate likelihood ratio test and Shimodaira-Hasegawa (aLRT-SH) used as an estimate for branch support in PhyML and the posterior probabilities shown for the Bayesian analysis. Again these are the same methods used by Mei and Dvornyk, with the exception that their alignment was trimmed manually using Bioedit, where we used Gblocks (Mei and Dvornyk 2015). The low stringency setting selected in Gblocks ensured that only isolated elements of particular sequences were removed. The LG (Le and Gascuel) model was used in all cases for tree generation, as chosen by smart model selection through the PhyML software (Le and Gascuel 2008).

The three resulting trees are presented in Figures $\mathrm{S} 1$ (MUSCLE), S2 (MAFFT) and S3 (PRANK). The previous analysis (Mei and Dvornyk 2015) aligned the sequences using MUSCLE. Hence we present the analysis based on MUSCLE here as our primary output. Our trees are presented unrooted, as is the case for trees from Lucas-Lledó and Lynch (2009) and Mei and Dvornyk (2015). The latter work devoted significant effort to establishing the geological timeline of the evolution of the photolyase/cryptochrome superfamily, giving confidence that CPD Class II Photolyases are basal to the superfamily. From our biochemical perspective, it is sensible to effectively use the CPD Class II Photolyases as an outgroup for the analysis, since, as discussed below, they only possess a "dyad" of tryptophan residues as compared with the triads and tetrads that are characteristic of the other subfamilies.

The (6-4) Photolyase/Animal Cry subfamily, the CryDASH subfamily and the Plant Photolyase subfamily appear in our Figures S1 and summary Fig. 2 much as they do in the equivalent tree from Mei and Dvornyk. The relative positions of the CPD Class I Photolyase and Plant Cry subfamilies show less consistency between our tree in Fig. 2 and Mei and Dvornyk's results. However, the tree based on our MAFFT alignment (Fig. S2) exhibits more similarity. We should also note that the literature phylogenetic tree that includes more bacterial sequences (Lucas-Lledó and Lynch 2009) places the CPD Class I Photolyase and Plant Cry subfamilies quite differently. We conclude that our findings both broadly accord with the literature and reflect the unresolved subtleties of the previous analyses.

We should note an interesting set of orthologues from Chloroflexi bacteria. These five proteins form a monophyletic group in all three of our trees (Figs. S1-S3), but are located differently in each case with respect to the six subfamilies. When these sequences are modelled they appear to be most similar to CPD Class III Photolyases (these sequences align best with $4 \mathrm{U} 63$ from the CPD Class III Agrobacterium tumefaciens structure with $42 \%$ sequence identity between Roseiflexus orthologue and $43.38 \%$ homology with Chlorflexus aggregans orthologue) (Scheerer et al. 2015).

\section{Incongruences in the Evolution of the Superfamily}

The occurrence of horizontal gene transfer from ancestral bacteria to ancestral eukaryotes is widely accepted to have resulted from the endosymbioses that formed mitochondria and also, in the case of plants, chloroplasts. More recently, further examples of possible HGT have started to emerge. Indeed Lucas-Lledó and Lynch have mentioned the importance of gene gain, by HGT, as well as gene loss during the evolution of the photolyase/cryptochrome superfamily (Lucas-Lledó and Lynch 2009). However, these authors did not explore in detail how such HGT events contribute to the 


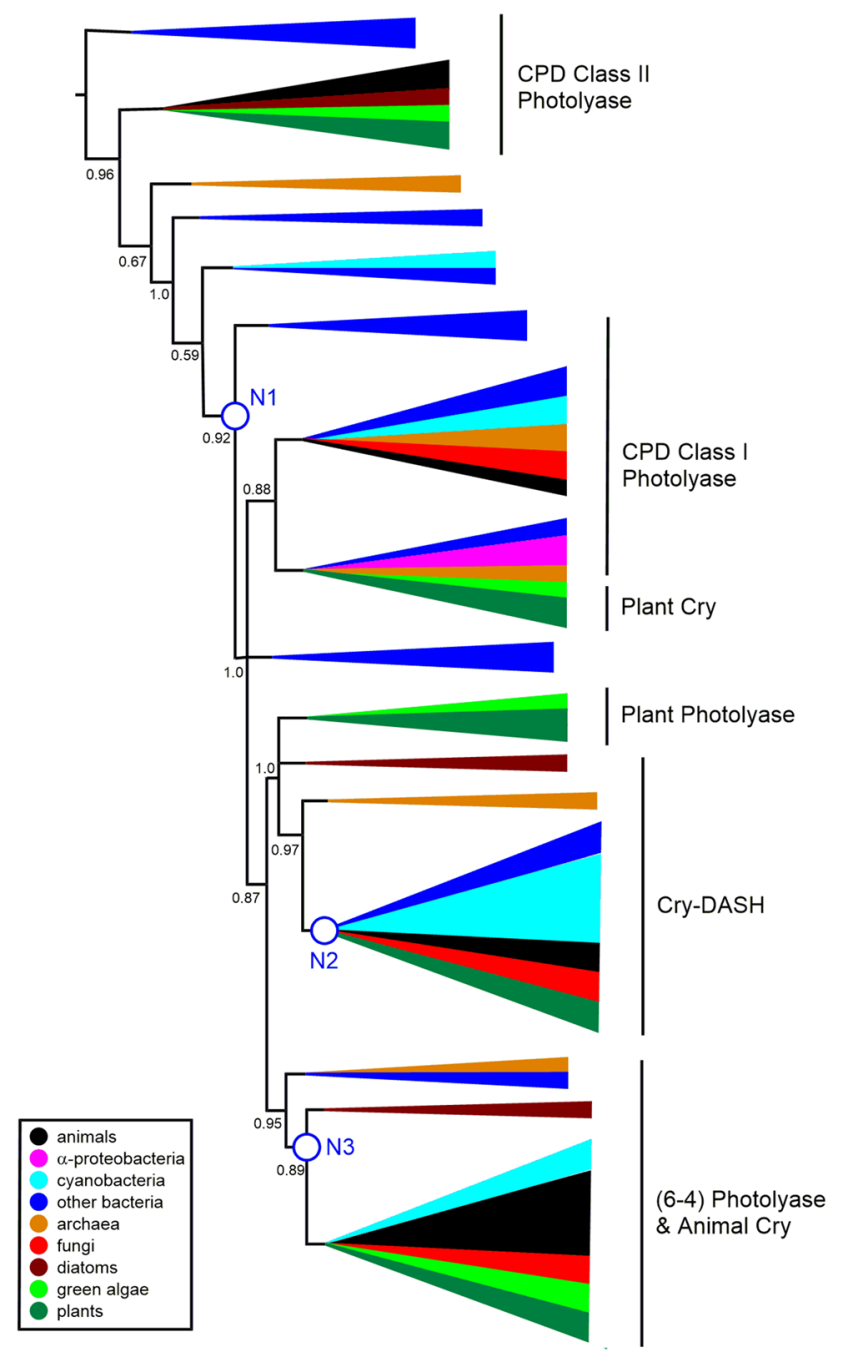

Fig. 2 Schematic phylogenetic tree from MUSCLE alignment. The full tree is in Supplementary Figure S1. aLRT-SH over 0.5 are shown, with branches below 0.5 being collapsed. We have not attempted to precisely define the different clades, but have indicated the locations of the main subfamilies with reference to Fig. 1 and Mei and Dvornyk (2015). N1-N3 refer to the three nodes discussed in the manuscript. The Figure highlights the positions of animal sequences (in black) relative to $\alpha$-proteobacterial sequences (in magenta) and cyanobacterial sequences (in cyan). Also colour coded are sequences from other bacteria (blue), archaea (orange), fungi (red), diatoms (brown), green algae (light green) and plants (dark green) (Color figure online)

acquisition of new function during evolution and this is our primary focus.

To identify putative HGT occurrences, we have followed guidance from authors including Husnik and McCutcheon (2018) who conclude that "Phylogenetic conflict (that is, incongruence of a single-gene tree with a known species phylogeny) is the method of choice for the detection of HGT events." We have identified three significant incongruences in our photolyase/cryptochrome "single-gene tree" that are visible in all three Figures S1-S3. The relevant nodes are annotated in summary Fig. 2 as N1, N2 and N3. Node 1 (N1) represents a common ancestor of photolyases and cryptochromes from various taxa of bacteria, from archaea, from algae, fungi, plants and animals. However, this appears more recently than the common ancestor of animal CPD Class II Photolyase proteins and the other animal paralogues in the superfamily. This is clearly incongruent with the standard tree of life. Node 2 (N2) represents the most recent common ancestor of Cry-DASH proteins from a group of eukaryotes, including animals, and of bacteria, including numerous cyanobacterial proteins. Again, the standard model would not predict this to fall more recently than the common ancestor of the eukaryotic members of the superfamily. Node 3 (N3) represents the most recent common ancestor of cryptochromes (Cry) from animals, fungi, plants, algae and, remarkably, two species of Gloeobacter, a further striking incongruence. This last feature was noted by Lucas-Lledó and Lynch (2009), though G. violaceus was the only Gloeobacter genome then available. They invoked a HGT from green algae to $G$. violaceus.

The trees from Lucas-Lledó and Lynch (2009) and Mei and Dvornyk (2015) show a node, similar to our Node 1, that represents a common ancestor of all cryptochrome/ photolyase proteins, except CPD Class II Photolyases, in most major phyla of bacteria and eukaryotes. They also both show a node, similar to our Node 2, that represents a common ancestor of (6-4) Photolyase/Animal Cry and bacterial Cry-DASH sequences (though Lucas-Lledó and Lynch did not label this group as Cry-DASH). Our tree is the first to include sequences from both sequenced species of Gloeobacter, but the location of Node 3 accords with the placement of $G$. violaceus in the literature trees. These previous authors did not discuss these apparent incongruences in detail.

We should also note the presence of archaeal sequences in our data set. Apart from one sequence from Thaumarchaeota, all the archaeal species represented are Euryarchaeota, in which HGT from bacteria is a common feature (see for example Santa-Molina et al. 2020). Therefore, the presence of photolyase/cryptochrome superfamily genes in these archaea is not informative with respect to the analysis presented here.

\section{Conserved Residues and Hence Functionalities are Observed Across All Families}

Previously Zhang et al (2017) identified conserved residues in the superfamily that relate to DNA binding within the photolyase, defining which substrate is recognised and repaired by the photolyase (Table 1 and Fig. 3). In Fig. 3 they are labelled as position 1 and position 2 . Looking at position 1 we can see that, like their eukaryotic counterparts, bacterial orthologues of CPD Photolyase proteins have a 
Table 1 Conserved residues identified across members of the superfamily

\begin{tabular}{llll}
\hline Subfamily & Position 1 & Position 2 & Substrate \\
\hline (6-4) photolyase & H & W & $6-4$ \\
Animal Cry & H & W & - \\
CPD class I & M & W & CPD \\
CPD class II & M & W & CPD \\
CPD class III & M & W & CPD \\
Cry-DASH & Q & Y & ssDNA \\
Plant Cry & V & Y & - \\
Plant photolyase & - & - & \\
\hline
\end{tabular}

methionine in this position. The cyanobacterial Gloeobacter sequences that surprisingly group with (6-4) Photolyase/ Animal Cry have the characteristic histidine at the same position and bacterial orthologues of Cry-DASH have the expected glutamine.

Conversely, in position 2, all of the members of the CPD Photolyase subfamilies have a tryptophan at this position apart from the fungal CPD Photolyase, which, in comparison to its bacterial orthologue, contains a tyrosine. The bacterial orthologues of Cry-DASH retain the tyrosine also at position 2. The Gloeobacter sequences again have the conserved tryptophan seen in the rest of the (6-4) Photolyase/Animal Cry subfamily. The bacterium Xanthomonas axonpodis that groups with the Plant Cry subfamily appears to have residues more like a CPD Photolyase at these positions (M358 and W397). The Plant Photolyase member doesn't convey any conserved residues at these positions. Conservation of these residues in the bacterial sequences in our study gives confidence that the orthologues are genuine and that they will have the same function in vivo as eukaryotic members of the same subfamily.

\section{The "Canonical" Tryptophan Triad and Tryptophan Tetrad Motifs have Emerged Since the Divergence with CPD Class II Photolyases}

Crucial to the function of some members of this superfamily is a triad of tryptophan residues that are required for the transmission of the electron from FAD to create FADH which is then, for example, used for the repair of DNA in (6-4) Photolyases (Hitomi et al. 2009). We mapped the presence of these three tryptophans on our phylogenetic tree and observe that this mechanism appears to have evolved more recently than the divergence from CPD Class II Photolyase proteins (Fig. 4). The three tryptophan residues are present in the majority of orthologues that have evolved since Node 1. In subsequent research, the tryptophan triad that is involved in electron transfer to FAD has been expanded to include a further tryptophan in Drosophila and Xenopus (Müller et al. 2015; Nohr et al. 2016; Yamamoto et al. 2017; Lin et al. 2018). This tryptophan "tetrad" increases the lifetime of FADH. The fourth tryptophan in the tetrad is replaced by a tyrosine in algae and plants, which is able to produce a similar effect (Nohr et al. 2016; Oldemeyer et al. 2016; Franz et al. 2018).

We wondered when this tryptophan tetrad motif evolved, so looked for the presence of the fourth tryptophan throughout our phylogenetic analysis. The sequences that contain this fourth tryptophan are shown on the phylogenetic tree in Fig. 4 with red branches. All of the animal cryptochromes have this fourth tryptophan, usually in a four amino acid motif of $W(\Phi) \mathrm{SW}$, where the first Trp is the newly identified member of the "tetrad". We also identified this motif in the two sequenced Gloeobacter, which are cyanobacteria (Gloeobacter kilaueensis WP_023172734.1 and Gloeobacter violaceus WP_011141747.1) (Figs. 5 and 6). These proteins group near the algal (6-4) Photolyases, which are

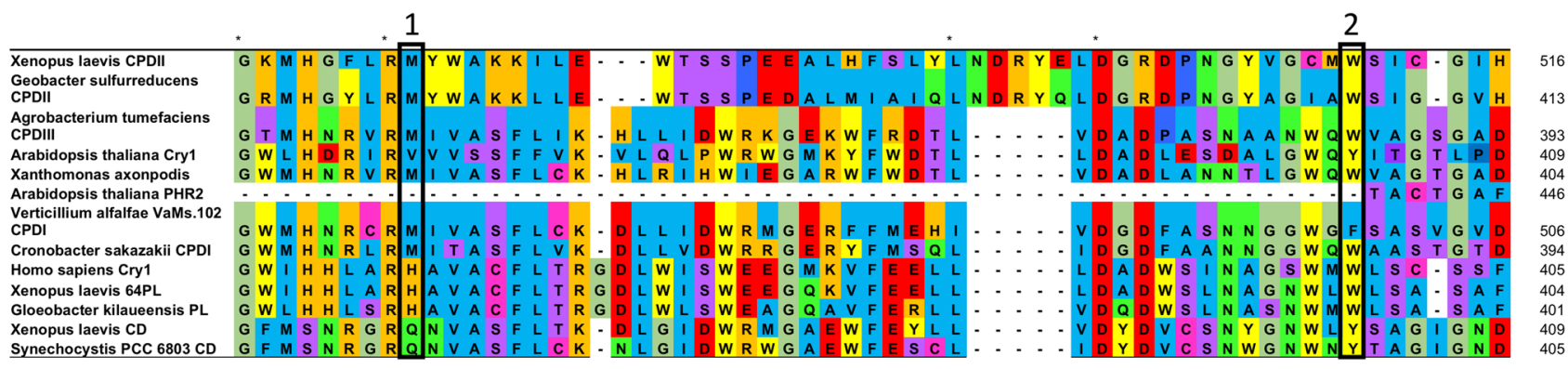

Fig. 3 Alignment of examples of each subfamily alongside bacterial orthologues for each (aside from CPD III which is characterised from the bacteria A. tumefaciens and from Plant Photolyase A. thaliana which has no bacterial orthologues). Residues involved in substrate recognition are highlighted with a black box and whole conserved residues are shown with an *. Sequences used are $X$. laevis (6-4) PL (NP_001081421.1), X. laevis CPD II (NP_001089127.1),
X. laevis CD (NP_001084438.1), Geobacter sulfurreducens CPD II (WP_010943456.1), Agrobacterium tumificans (WP_010971478.1), A. thaliana Cry1 (NP_567341.1), A. thaliana PHR2 (NP_182281.1), X. axonpodis (WP_011050942.1), V. alfalfae (XP_002999933.1), Cronobacter sakazakii WP_012125345.1, H. sapiens Cry1 (NP_004066.1), G. kilaueensis (WP_023172734.1), Synechocystis PCC 6803 CD (WP_014407097.1) (Color figure online) 


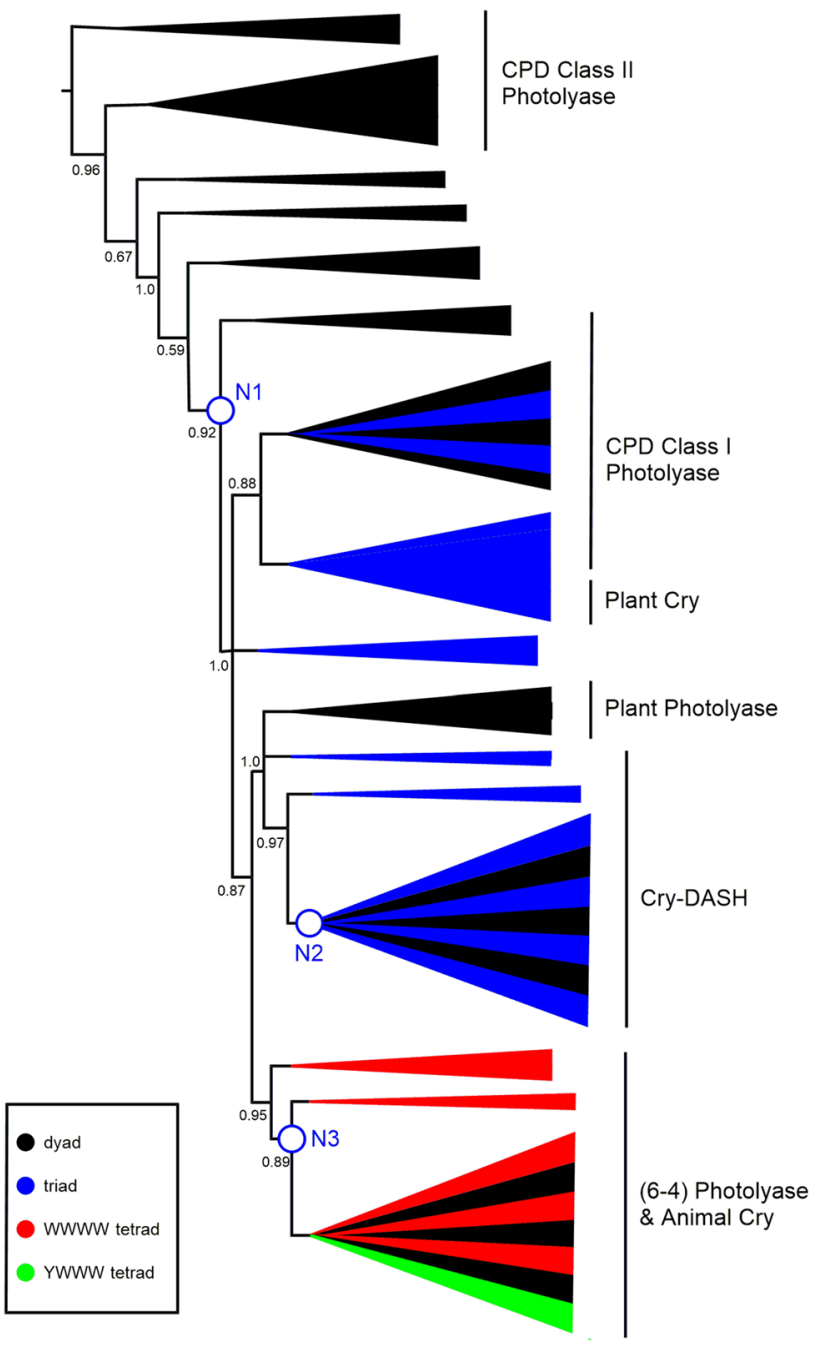

Fig. 4 Schematic phylogenetic tree from MUSCLE alignment. aLRT-SH over 0.5 are shown, with branches below 0.5 being collapsed. N1-N3 refer to the three nodes discussed in the manuscript. Proteins that contain the "canonical" tryptophan triad are represented by blue branches. Proteins with a WWWW tetrad are represented by red branches and with a YWWW tetrad green. The canonical triad is found widely through the CPD Class I, Plant Cry and Cry-DASH subfamilies. Tetrads are found mainly in the (6-4) Photolyase/Animal Cry subfamily. The full tree is in Supplementary Figure S4 (Color figure online)

able to undertake DNA repair and gene control using an unusual arrangement of long C-terminal extension (Kottke et al. 2017; Franz et al. 2018). As mentioned above, the algal proteins mainly have a $Y(\Phi)$ SW motif instead of $W(\Phi) \mathrm{SW}$ and hence a YWWW version of the WWWW tetrad.

We should note that CPD Class II Photolyases use a different set of tryptophans from the rest of the classes. This tryptophan 'dyad' was defined by Kiontke et al. (2011). All of the CPD Class II Photolyase orthologues in this analysis contain these two tryptophans (numbered W449 and W470 in Xenopus laevis CPD II). These proteins also contain a conserved tyrosine, suggesting that the electron transfer in this subfamily differs from the usual all tryptophan mechanism (Y434 in X. laevis CPD II). As mentioned earlier, this provides a firm biochemical justification for treating CPD Class II Photolyases as, essentially, an outgroup in our phylogenetic analysis.

\section{An "Alternative" Tryptophan Triad is Characteristic of Cry-DASH Proteins}

Whilst the canonical triad (and tetrad) of tryptophan residues is characteristic of all subfamilies of the photolyase/ cryptochrome superfamily except CPD Class II Photolyases, experiments in Escherichia coli and Arabidopsis thaliana have shown that this particular triad is not always essential for function (Y. F. Li et al. 1991; X. Li et al. 2011). Indeed, Biskup et al. (2011) showed in the cyanobacterium Synechocystis that an "alternative" triad of tryptophan residues could mediate electron transfer (Fig. 6b). Two of the canonical tryptophan residues still contribute to the alternative set, but in Synechocystis a W320 completes the triad, not W375. In some orthologues, the redundant tryptophan is lost.

In Figures S5 and S7, sequences containing the "alternative" triad of tryptophan residues are indicated with green coloured branches (Fig. 7). Importantly, the identification of the different triads in our work rests solely on the alignment of the sequences. In many cases, the proteins possess the elements of both the canonical and alternative triads and, with the exception of Synechocystis (see above), we do not know which of them is functional. The alternative triad is found sporadically amongst CPD Class I Photolyases, including those that use a different cofactor (5,10-methylenetetrahydrofolate, MTHF) and that are often called CPD Class III Photolyases (Scheerer et al. 2015). The alternative pattern is not found at all in Plant Photolyase proteins, nor in (6-4) Photolyase/Animal Cry.

Strikingly, the alternative tryptophan triad appears to be an essential feature of Cry-DASH proteins, being present in all the Cry-DASH sequences in our phylogenetic tree, with the exception of the proteins from diatoms Thalassiosira pseudodonna and Phaeodactylum tricornutum (Figure S5). In fact, the P. tricornutum protein has been shown experimentally to more closely resemble a Plant Photolyase in function, hence the annotation as Cry-DASH could be revisited (Juhas et al. 2014).

\section{Discussion}

We present here a phylogenetic analysis of cryptochrome and photolyase proteins that includes an array of prokaryotic sequences. The tree in Fig. 2 has all the hallmarks of the previous definitive analysis of this superfamily from Mei 


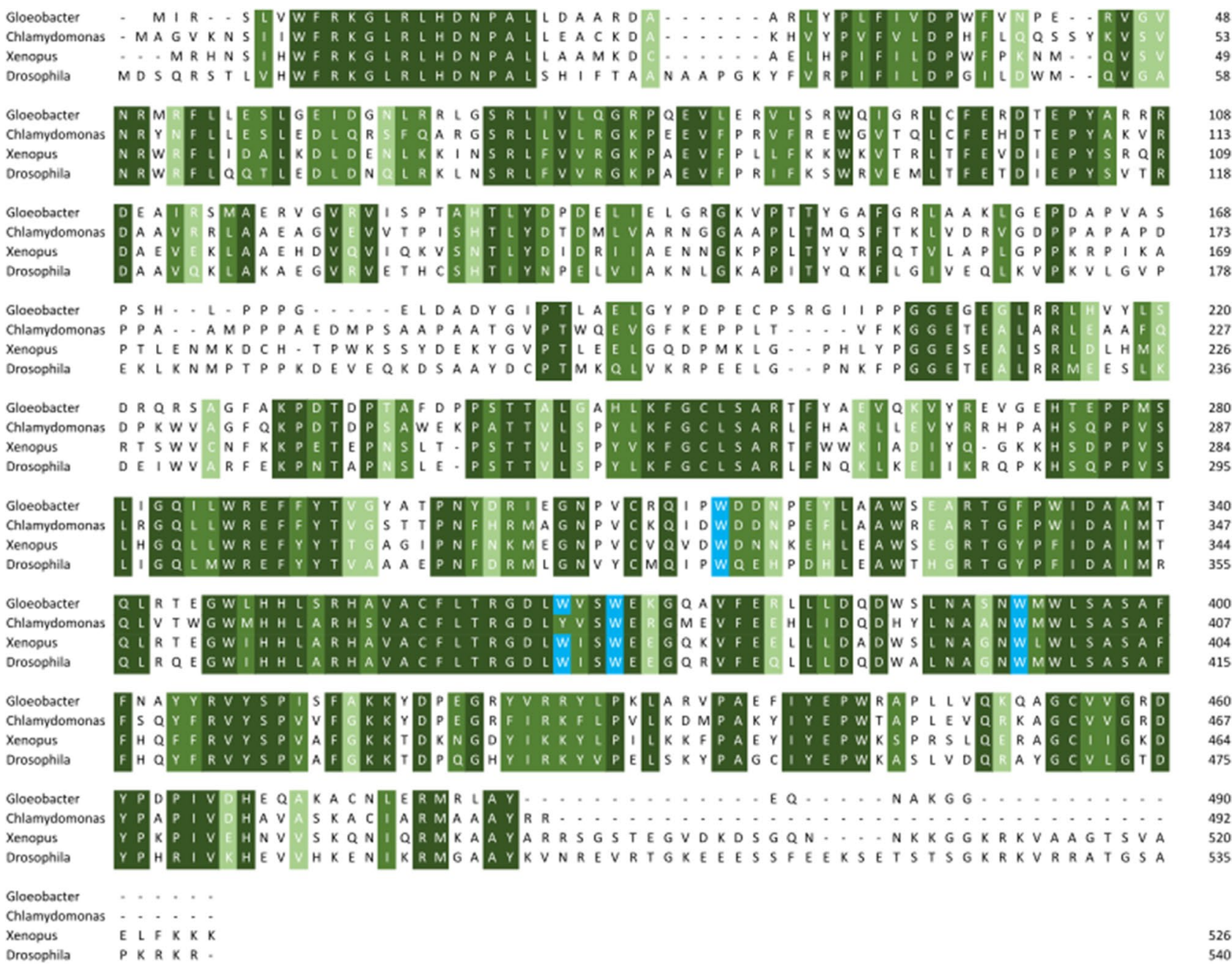

Fig. 5 Alignment of (6-4) Photolyase orthologues. Gloeobacter violaceus (6-4) PL (WP_011141747.1), X. laevis (6-4) PL (NP_001081421.1), Chlamydomonas reinhardtii (6-4) PL (XP_001698054.1) and Drosophila melanogaster (6-4) PL (BAA12067.1). Residues in the tryptophan tetrad are highlighted in

and Dvornyk. However, similar to our previous research on retinoic acid biosynthesis (Miles et al. 2019; Millard et al. 2014), we find that inclusion of photolyase/cryptochrome sequences and candidate orthologues from across the kingdoms of life brings to the fore phylogenetic relationships that are particularly relevant to eukaryogenesis, in particular with respect to the placement of animal and bacterial orthologues.

We have highlighted three striking incongruences between our gene tree for the photolyase/cryptochrome superfamily and the widely accepted species tree that is based on analysis of ribosomal protein sequences (Hug et al. 2016). The simplest hypothesis that accords with this observation is to propose at least three HGT events between ancestral bacteria and ancestors of eukaryotes that have contributed to the emergence of this superfamily of proteins. blue, with the degree of conservation between sites shown in green, with darkest green being entirely conserved at that position. Gloeobacter is 50\% identical to the amphibian Xenopus, 50\% to the insect Drosophila, $56 \%$ to the alga Chlamydomonas (Color figure online)

Mapping the occurrence of tryptophan dyads, canonical and alternative triads and tetrads across the phylogenetic tree is revealing. Broadly, we can say that CPD class II photolyase proteins possess a dyad and are thus predicted to be the least effective at stabilising diradicals. Indeed, we are essentially using the CPD Class II Photolyase sequences as an outgroup in this study. We then see acquisition of triad motifs that approximately coincides with the putative HGT at Node 1. Strikingly, within the Cry-DASH subfamily that has a common ancestor at Node 2, we find that the "alternative" triad is completely conserved, again indicating this feature may have accompanied HGT. Finally, around Node 3 , where we propose a further HGT, we see appearance of tryptophan tetrad motifs. These tryptophan signatures provide powerful additional support for multiple HGT events. 
A

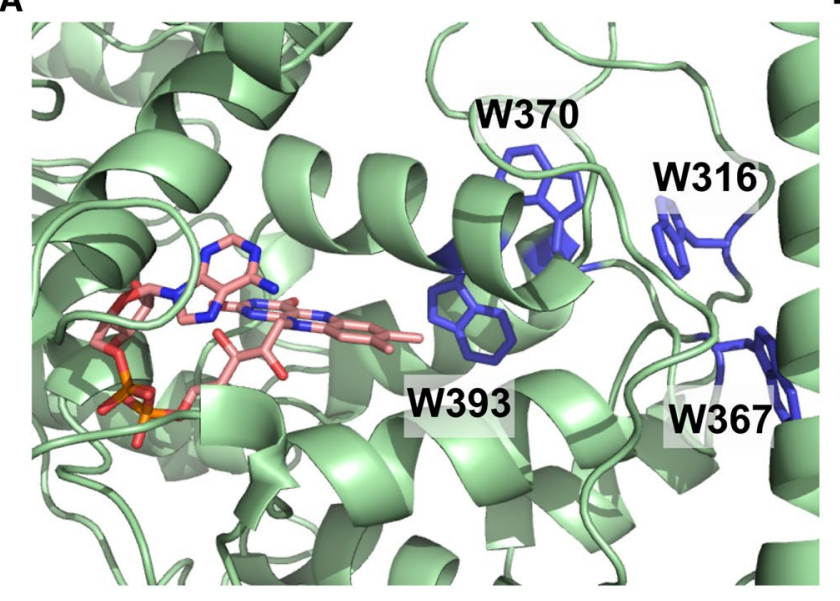

B

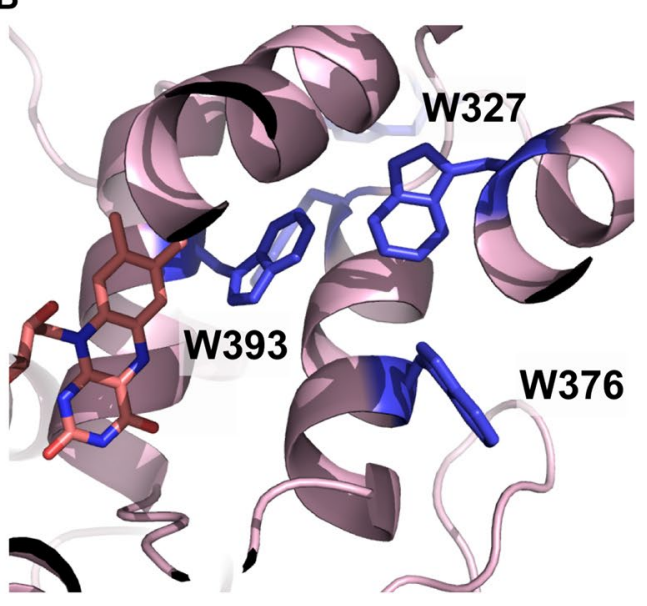

Fig. 6 a Model of (6-4) Photolyase orthologue from G. kilaueensis using Phyre 2. The conserved tryptophan tetrad is shown in blue with FAD modelled in from 3FY4 shown in peach. b Model of a Cry-
DASH orthologue from G. kilaueensis with the "alternative" tryptophan triad shown in blue (Color figure online)

with cyanobacteria than with $\alpha$-proteobacteria (see Node 2). Gabaldón (2018) and López-García and Moreira (2020) argue that is very difficult to reconcile such observations with just an EGT from an $\alpha$-proteobacterium to form the mitochondrion. We would need to assume that orthologues of Animal Cry and of animal Cry-DASH genes had been lost in all sequenced extant species of $\alpha$-proteobacterium (with the exception of those mentioned above where there are "distant" orthologues). Rather, evidence is accumulating for "waves" of acquisition of genes from different bacterial sources in addition to the endosymbiosis to form the mitochondrion and Gabaldón (2018) says "we need to go beyond simple models that involve a single bacterial endosymbiont engulfed by an archaeal ancestor".

Intriguingly, the two Gloeobacter species in our analysis have photolyase/cryptochrome proteins that exhibit all of the canonical triad, the alternative triad and the tetrad of tryptophan residues (Fig. 8). The Gloeobacter proteins with the tryptophan tetrad are annotated as (6-4) Photolyases in both $G$. kilauensis and $G$. violaceus and the proteins with the characteristic alternative triad are annotated, consistent with our observation on the Cry-DASH group above, as CryDASH in both species. A further pair of Gloeobacter proteins group with a number of CPD Class I Photolyases that are believed to use 8-HDF as the second cofactor. Finally, $G$. kilauensis has yet another, unannotated, paralogue.

Gloeobacter species are generally accepted to have branched from the rest of the cyanobacteria very early in evolutionary history (Schirrmeister et al. 2013). Gloeobacter species lack thylakoid membranes that are the normal sites in cyanobacteria of light-dependent reactions. G. kilaueensis was discovered in a Hawaiian lava cave in 2013 (Saw et al. 2013), whilst G. violaceus PCC 7421 was isolated from a 


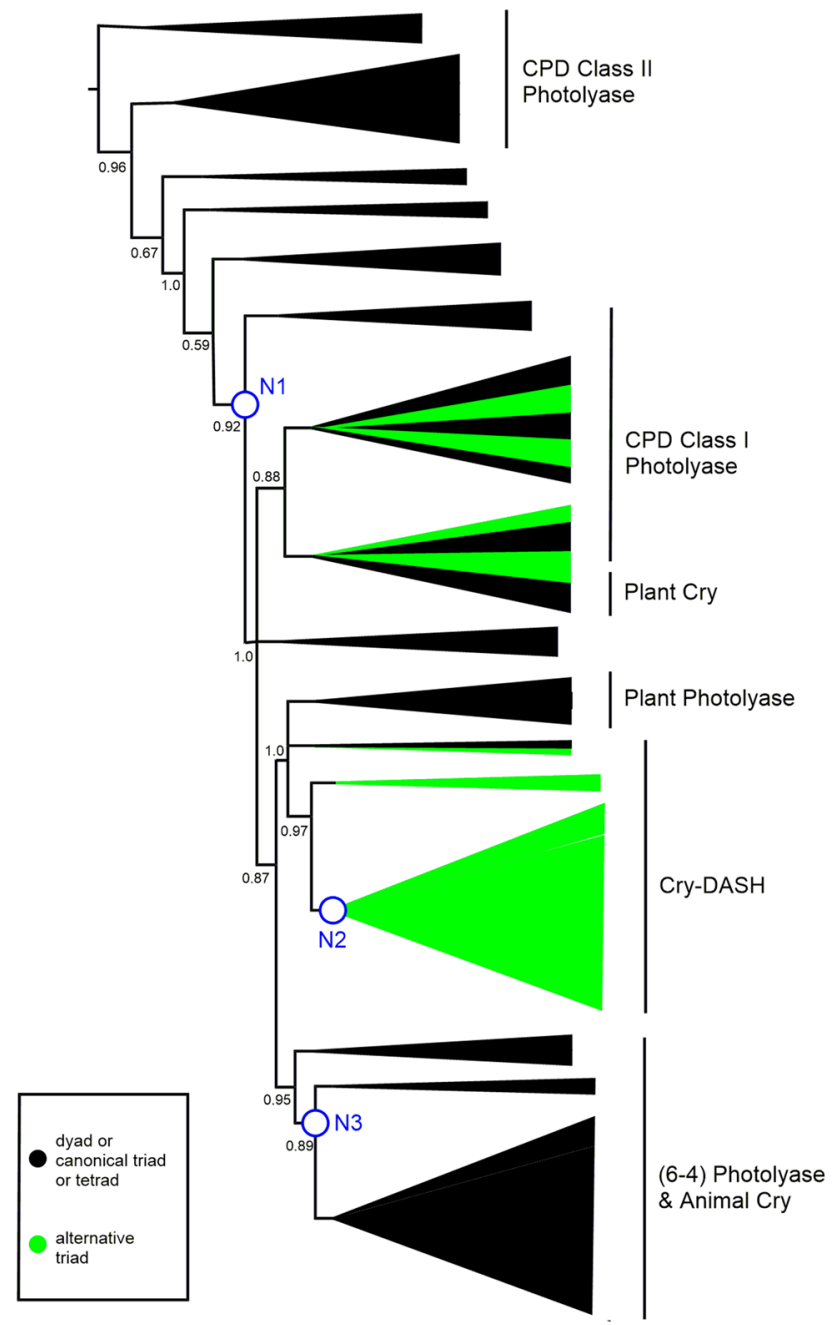

Fig. 7 Schematic phylogenetic tree from MUSCLE alignment. aLRTSH over 0.5 are shown, with branches below 0.5 being collapsed. N1-N3 refer to the three nodes discussed in the manuscript. Clades with proteins that exhibit the "alternative" tryptophan triad are highlighted either in green, where almost all examples have the alternative triad (Cry-DASH), or green and black where some but not all examples have the alternative triad, as in the CPD Class I Photolyase and Plant Cry subfamilies. The full tree is in Supplementary Figure S5 (Color figure online)

limestone rock surface (Nakamura et al. 2003; Mareš et al. 2013). To confirm that the seven photolyase/cryptochromes all most probably evolved as paralogues in Gloeobacter, the GC contents of the genes were compared with that of the overall organism. All seven of the photolyase/cryptochrome genes in Gloeobacter have GC contents that are consistent with that of the whole organism (Table 2), which is evidence against acquisition of these genes by a more recent HGT to the cyanobacteria, as proposed by Lucas-Lledó and Lynch (2009).

Overall, our analysis suggests that bacteria have contributed significantly and repeatedly to the evolution of photolyases and cryptochromes across all kingdoms of life, including in animals, by putative HGT. These apparent gene transfer events seem to be linked to the acquisition of additional and/or different tryptophan residues that contribute to increased stability of the diradical intermediate that is so crucial to the mechanism of photoreception. We are mindful of the dangers of inferring features of species trees from single gene trees (Ku et al. 2015) and that is not our intention. Rather we aim to show that incongruent nodes in this gene tree align with fundamental distinctions in the biochemistry of different groups of photolyase/cryptochrome proteins, which invites further investigation. Indeed, case studies such as the complex phylogeny of the photolyase/cryptochrome superfamily could contribute valuable insight to emerging models of eukaryogenesis that acknowledge a rich bacterial heritage.

\section{Materials and Methods}

\section{Phylogenetic Analyses}

Cryptochrome and photolyase starting sequences were chosen from (Mei and Dvornyk 2015) and BLASTp searched against the NCBI non-redundant protein database against bacteria with the default settings to identify orthologous proteins. The initial sequences were Animal CRY (Homo sapiens NP_004066 and NP_066940), (6-4) Photolyase ( $X$. laevis NP_001081421), CPD Class II Photolyase (Monosiga brevicollis MX1 XP_001746666), Cry-DASH (Salpingoeca sp. ATCC 50,818 XP_004989008), Fungal CPD Class I Photolyase (Verticillium alfalfae VaMs.102 XP_002999933); Plant Cry (A. thaliana NP_567341 and NP_171935); Plant Photolyase (A. thaliana NP_182281). The top 20 bacterial orthologues for all subfamilies were chosen, along with orthologues from all other groups of life. Full details of sequences collected are in the Supplementary Information. Some sequences were removed when they were found to be from incomplete genome sequences such as those derived from metagenomes.

Amino acid sequences were aligned using MAFFT with method FFT-NS-I (Katoh et al. 2017), MUSCLE (Edgar 2004) and PRANK (Löytynoja and Goldman 2010). Redundant regions of the alignment were then removed using Gblocks with low stringency settings (Minimum number of seq for a conserved and flank position set to half, Maximum number of contiguous nonconserved positions: 50, Minimum length of a block: 2, All gap positions allowed) (Castresana 2000). This retained 739 positions out of 2049 with the MUSCLE alignment (36\% in 5 blocks), 732 positions out of 2075 with the MAFFT alignment (38\% in 6 blocks) and 697 positions out of 5842 in the PRANK alignment (12\% in 10 blocks). Phylogenetic trees were then built using PhyML 
Fig. 8 All four photolyase/ cryptochrome family members from G. kilaueensis modelled with conserved tryptophans shown. Green-(6-4) Photolyase that contains the fourth tryptophan, Blue-CPD Class II like orthologue that comprises a tryptophan dyad, Pink-CryDASH. Orange - unannotated paralogue. All structures were modelled using Phyre 2.0 (Color figure online)

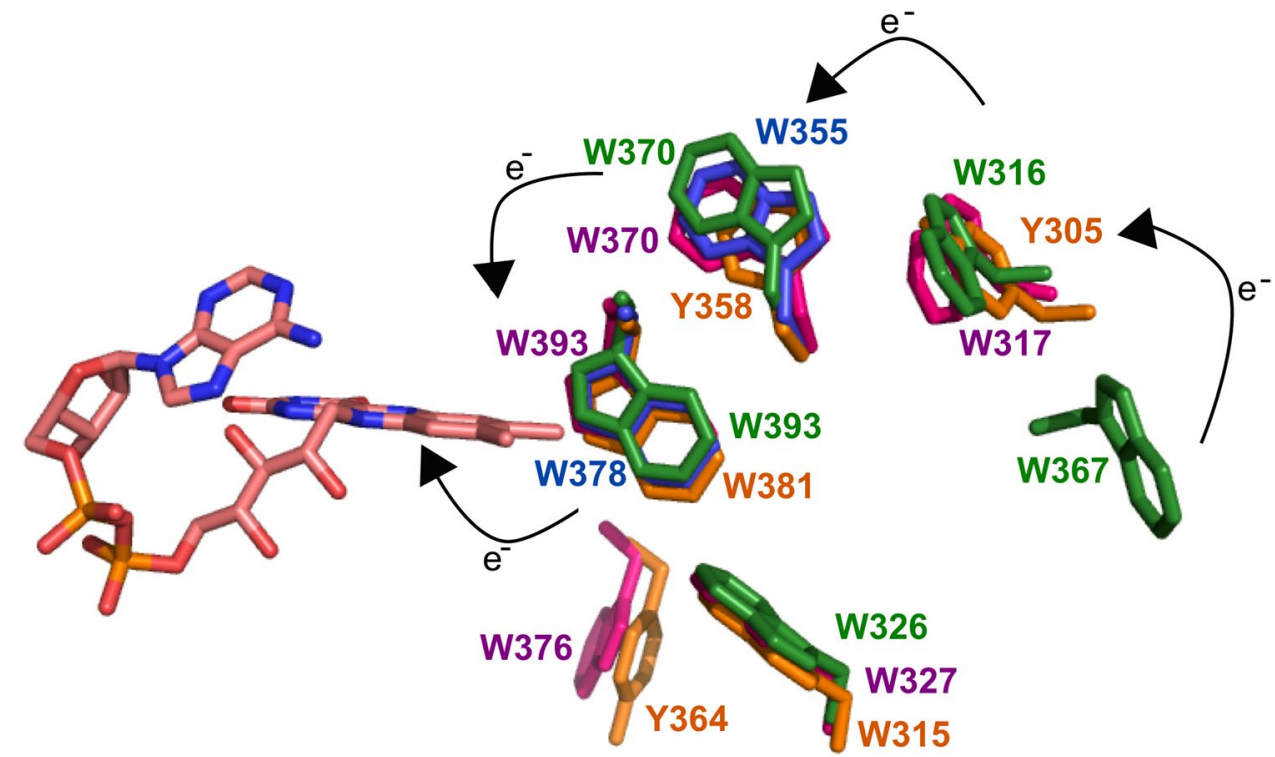

Table 2 The GC content of orthologues identified in Gloeobacter compared to the GC content of the complete genome

\begin{tabular}{|c|c|c|c|c|c|}
\hline Organism & $\begin{array}{l}\text { Annotation in phylogenetic } \\
\text { analysis }\end{array}$ & Genome content & Gene content & Accession code & Closest orthologue \\
\hline \multirow[t]{4}{*}{ Gloeobacter kilaueensis JS1 } & Gloeobacter_kilaueensis & 60.5 & 60.89 & WP_023175030.1 & CPD Class III \\
\hline & Gloeobacter_kilaueensis_DASH & & 62.97 & WP_023173156.1 & Cry-DASH \\
\hline & Gloeobacter_kilaueensis_PL & & 62.74 & WP_023172734.1 & (6-4) Photolyase \\
\hline & Gloeobacter_kilaueensis_PL2 & & 63.45 & WP_023172026.1 & CPD \\
\hline \multirow[t]{3}{*}{ Gloeobacter violaceus PCC 7412} & Gloeobacter_violaceus & 62 & 62.3 & WP_011141747.1 & (6-4) Photolyase \\
\hline & Gloeobacter_violaceus_CPD & & 64.64 & WP_011140786.1 & CPD \\
\hline & Gloeobacter_violaceus_DASH & & 60.733 & WP_011140837.1 & Cry-DASH \\
\hline
\end{tabular}

with approximate likelihood ratio testing giving branch support values and the Le and Gascuel (LG) model used which had been chosen in Smart Model Selection (Le and Gascuel 2008; Guindon et al. 2010; Lefort et al. 2017). Trees were analysed and annotated using Treegraph2 (Stöver and Müller 2010).

\section{GC Content Calculation}

Genomes in the NCBI were used to identify the GC content of the entire genome of a bacteria and compare this to the GC content of the gene. A disparency of $8 \%$ was the cutoff set for a gene to be 'real' and not a possible contaminant.

\section{Modelling Cyanobacterial Proteins}

The structure of G. kilaueensis (WP_023172734.1) and G. violaceus (WP_011141747.1) (6-4) PL were modelled using Phyre 2.0 (Kelley et al. 2015) with 3FY4 as the template (Hitomi et al. 2009). The Cry-DASH orthologues were modelled with $1 \mathrm{NP} 7$ as the template $(G$. kilaueensis WP_023173156.1 and G. violaceus WP_011140837.1) (Brudler et al. 2003). The 8-HDF like orthologues were modelled with $1 \mathrm{TEZ}$ as the template (G. kilaueensis WP_023172026.1 and G. violaceus WP_011140786.1) (Mees et al. 2004). Finally, the other G. kilaueensis orthologue was modelled with $4 \mathrm{U} 63$ as a template (WP_023175030.1) (Scheerer et al. 2015).

Acknowledgements This work was undertaken on MARC1, part of the High Performance Computing and Leeds Institute for Data Analytics (LIDA) facilities at the University of Leeds, UK. Martin Callaghan from the HPC at University of Leeds for help with establishing methods. We also thank Professor Mary O'Connell from the University of Nottingham for her input during the development of this project.

Funding Open access funding provided by University of Leeds.

Data Availability Data provided as Supplementary Information. 


\section{Compliance with Ethical Standards}

Conflict of interest Paul Taylor is a Director of Tangent Reprofiling Limited. Other authors have no competing financial interests.

Open Access This article is licensed under a Creative Commons Attribution 4.0 International License, which permits use, sharing, adaptation, distribution and reproduction in any medium or format, as long as you give appropriate credit to the original author(s) and the source, provide a link to the Creative Commons licence, and indicate if changes were made. The images or other third party material in this article are included in the article's Creative Commons licence, unless indicated otherwise in a credit line to the material. If material is not included in the article's Creative Commons licence and your intended use is not permitted by statutory regulation or exceeds the permitted use, you will need to obtain permission directly from the copyright holder. To view a copy of this licence, visit http://creativecommons.org/licenses/by/4.0/.

\section{References}

Biskup T, Hitomi K, Getzoff ED et al (2011) Unexpected electron transfer in cryptochrome identified by time-resolved EPR spectroscopy. Angew Chemie - Int Ed. https://doi.org/10.1002/ anie. 201104321

Brash AR, Niraula NP, Boeglin WE, Mashhadi Z (2014) An ancient relative of cyclooxygenase in cyanobacteria is a linoleate 10S-dioxygenase that works in tandem with a catalase-related protein with specific 10S-hydroperoxide lyase activity. J Biol Chem. https://doi.org/10.1074/jbc.M114.555904

Brudler R, Hitomi K, Daiyasu H et al (2003) Identification of a new cryptochrome class. Mol Cell. https://doi.org/10.1016/S1097 $-2765(03) 00008-X$

Cailliez F, Müller P, Firmino T et al (2016) Energetics of photoinduced charge migration within the tryptophan tetrad of an animal (6-4) photolyase. J Am Chem Soc. https://doi.org/10.1021/ jacs.5b10938

Cashmore AR, Jarillo JA, Wu YJ, Liu D (1999) Cryptochromes: blue light receptors for plants and animals. Science. https://doi. org/10.1126/science.284.5415.760

Castresana J (2000) Selection of conserved blocks from multiple alignments for their use in phylogenetic analysis. Mol Biol Evol. https://doi.org/10.1093/oxfordjournals.molbev.a026334

Edgar RC (2004) MUSCLE: multiple sequence alignment with high accuracy and high throughput. Nucleic Acids Res. https://doi. org/10.1093/nar/gkh340

Franz S, Ignatz E, Wenzel S et al (2018) Structure of the bifunctional cryptochrome aCRY from Chlamydomonas reinhardtii. Nucleic Acids Res. https://doi.org/10.1093/nar/gky621

Gabaldón T (2018) Relative timing of mitochondrial endosymbiosis and the "pre-mitochondrial symbioses" hypothesis. IUBMB Life. https://doi.org/10.1002/iub.1950

Guindon S, Dufayard JF, Lefort V et al (2010) New algorithms and methods to estimate maximum-likelihood phylogenies: assessing the performance of PhyML 3.0. Syst Biol. https://doi. org/10.1093/sysbio/syq010

Hitomi K, DiTacchio L, Arvai AS et al (2009) Functional motifs in the (6-4) photolyase crystal structure make a comparative framework for DNA repair photolyases and clock cryptochromes. Proc Natl Acad Sci. https://doi.org/10.1073/pnas.0809180106

Hore PJ, Mouritsen H (2016) The radical-pair mechanism of magnetoreception. Annu Rev Biophys. https://doi.org/10.1146/annur ev-biophys-032116-094545
Hug LA, Baker BJ, Anantharaman K et al (2016) A new view of the tree of life. Nat Microbiol. https://doi.org/10.1038/nmicr obiol.2016.48

Husnik F, McCutcheon JP (2018) Functional horizontal gene transfer from bacteria to eukaryotes. Nat Rev Microbiol. https://doi. org/10.1038/nrmicro.2017.137

Juhas M, Von Zadow A, Spexard M et al (2014) A novel cryptochrome in the diatom Phaeodactylum tricornutum influences the regulation of light-harvesting protein levels. FEBS J. https ://doi.org/10.1111/febs. 12782

Kanai S, Kikuno R, Toh H et al (1997) Molecular evolution of the photolyase-blue-light photoreceptor family. J Mol Evol. https:// doi.org/10.1007/PL00006258

Katoh K, Rozewicki J, Yamada KD (2017) MAFFT online service: multiple sequence alignment, interactive sequence choice and visualization. Brief Bioinform. https://doi.org/10.1093/bib/bbx108

Kelley LA, Mezulis S, Yates CM et al (2015) The Phyre2 web portal for protein modeling, prediction and analysis. Nat Protoc. https ://doi.org/10.1038/nprot.2015.053

Kiontke S, Geisselbrecht Y, Pokorny R et al (2011) Crystal structures of an archaeal class II DNA photolyase and its complex with UV-damaged duplex DNA. EMBO J. https://doi.org/10.1038/ emboj.2011.313

Kiontke S, Gnau P, Haselsberger R et al (2014) Structural and evolutionary aspects of antenna chromophore usage by class II photolyases. J Biol Chem. https://doi.org/10.1074/jbc.M113.542431

Kottke T, Oldemeyer S, Wenzel S et al (2017) Cryptochrome photoreceptors in green algae: unexpected versatility of mechanisms and functions. J Plant Physiol. https://doi.org/10.1016/j.jplph .2017.05.021

Ku C, Nelson-Sathi S, Roettger M et al (2015) Endosymbiotic origin and differential loss of eukaryotic genes. Nature. https://doi. org/10.1038/nature14963

Le SQ, Gascuel O (2008) An improved general amino acid replacement matrix. Mol Biol Evol. https://doi.org/10.1093/molbev/ msn067

Lefort V, Longueville JE, Gascuel O (2017) SMS: smart model selection in PhyML. Mol Biol Evol. https://doi.org/10.1093/molbev/ msx 149

Li YF, Heelis PF, Sancar A (1991) Active site of DNA photolyase: tryptophan-306 is the intrinsic hydrogen atom donor essential for Flavin radical photoreduction and DNA repair in vitro. Biochemistry. https://doi.org/10.1021/bi00239a034

Li X, Wang Q, Yu X et al (2011) Arabidopsis cryptochrome 2 (CRY2) functions by the photoactivation mechanism distinct from the tryptophan (trp) triad-dependent photoreduction. Proc Natl Acad Sci. https://doi.org/10.1073/pnas.1114579108

Lin C, Top D, Manahan CC et al (2018) Circadian clock activity of cryptochrome relies on tryptophan-mediated photoreduction. Proc Natl Acad Sci. https://doi.org/10.1073/pnas.1719376115

López-García P, Moreira D (2019) Eukaryogenesis, a syntrophy affair. Nat Microbiol. https://doi.org/10.1038/s41564-019-0495-5

López-García P, Moreira D (2020) The Syntrophy hypothesis for the origin of eukaryotes revisited. Nat Microbiol. https://doi. org/10.1038/s41564-020-0710-4

Löytynoja A, Goldman N (2010) WebPRANK: a phylogeny-aware multiple sequence aligner with interactive alignment browser. BMC Bioinform. https://doi.org/10.1186/1471-2105-11-579

Lucas-Lledó JI, Lynch M (2009) Evolution of mutation rates: phylogenomic analysis of the photolyase/cryptochrome family. Mol Biol Evol. https://doi.org/10.1093/molbev/msp029

Magnani F, Nenci S, Millana Fananas E et al (2017) Crystal structures and atomic model of NADPH oxidase. Proc Natl Acad Sci. https ://doi.org/10.1073/pnas.1702293114

Mareš J, Hrouzek P, Kaňa R et al (2013) The primitive thylakoidless cyanobacterium Gloeobacter is a common rock-dwelling 
organism. PLoS ONE. https://doi.org/10.1371/journal.pone.00663 23

Mees A, Klar T, Gnau P et al (2004) Crystal structure of a photolyase bound to a CPD-like DNA lesion after in situ repair. Science. https ://doi.org/10.1126/science.1101598

Mei Q, Dvornyk V (2015) Evolutionary history of the photolyase/ cryptochrome superfamily in eukaryotes. PLoS ONE. https://doi. org/10.1371/journal.pone.0135940

Miles JA, Machattou P, Nevin-Jones D et al (2019) Identification of a cyanobacterial aldehyde dehydrogenase that produces retinoic acid in vitro. Biochem Biophys Res Commun. https://doi. org/10.1016/j.bbrc.2018.12.171

Millard A, Scanlan DJ, Gallagher C et al (2014) Unexpected evolutionary proximity of eukaryotic and cyanobacterial enzymes responsible for biosynthesis of retinoic acid and its oxidation. Mol Biosyst. https://doi.org/10.1039/c3mb70447e

Müller P, Yamamoto J, Martin R et al (2015) Discovery and functional analysis of a 4th electron-transferring tryptophan conserved exclusively in animal cryptochromes and (6-4) photolyases. Chem Commun. https://doi.org/10.1039/c5cc06276d

Nakamura Y, Kaneko T, Sato S et al (2003) Complete genome structure of Gloeobacter violaceus PCC 7421, a cyanobacterium that lacks thylakoids (supplement). DNA Res. https://doi.org/10.1093/dnare s/10.4.137

Nohr D, Franz S, Rodriguez R et al (2016) Extended electron-transfer in animal cryptochromes mediated by a tetrad of aromatic amino acids. Biophys J. https://doi.org/10.1016/j.bpj.2016.06.009

Oldemeyer S, Franz S, Wenzel S et al (2016) Essential role of an unusually long-lived tyrosyl radical in the response to red light of the animal-like cryptochrome acry. J Biol Chem. https://doi. org/10.1074/jbc.M116.726976

Öztürk N, Song SH, Özgür S et al (2007) Structure and function of animal cryptochromes. Cold Spring Harbor Symposia on quantitative biology. Cold Spring Harbor Laboratory Press, New York. https ://doi.org/10.1101/sqb.2007.72.015

Paps J (2018) What makes an animal? The molecular quest for the origin of the Animal Kingdom. Integr Comp Biol. https://doi. org/10.1093/icb/icy036

Picciano AL, Crane BR (2019) A nitric oxide synthase-like protein from Synechococcus produces $\mathrm{NO} / \mathrm{NO}_{3}{ }^{-}$from $\mathrm{L}$-arginine and NAPDH in a tetrahydrobiopterin- and $\mathrm{Ca}^{2+-}$ dependent manner. J Biol Chem. https://doi.org/10.1074/jbc.RA119.008399
Pittis AA, Gabaldón T (2016) Late acquisition of mitochondria by a host with chimaeric prokaryotic ancestry. Nature. https://doi. org/10.1038/nature 16941

Ponting CP, Aravind L, Schultz J, Bork P, Koonin EV (1999) Eukaryotic signalling domain homologues in archaea and bacteria. Ancient ancestry and horizontal gene transfer. J Mol Biol. https ://doi.org/10.1006/jmbi.1999.2827

Rawlings ND (2015) Bacterial calpains and the evolution of the calpain (C2) family of peptidases. Biol Direct. https://doi.org/10.1186/ s13062-015-0095-0

Santa-Molina C, Rivas-Marin E, Rojas AM et al (2020) Origin and evolution of polycyclic triterpene synthesis. Mol Biol Evol. https ://doi.org/10.1093/molbev/msaa054

Saw JHW, Schatz M, Brown MV et al (2013) Cultivation and complete genome sequencing of Gloeobacter kilaueensis sp. Nov., from a Lava Cave in Kîlauea Caldera, Hawai'I. PLoS ONE. https://doi. org/10.1371/journal.pone.0076376

Sancar A (2016) Mechanisms of DNA repair by photolyase and excision nuclease (nobel lecture). Angew Chem - Int Ed. https://doi. org/10.1002/anie.201601524

Scheerer P, Zhang F, Kalms J et al (2015) The class III cyclobutane pyrimidine dimer photolyase structure reveals a new antenna chromophore binding site and alternative photoreduction pathways. J Biol Chem. https://doi.org/10.1074/jbc.M115.637868

Schirrmeister BE, de Vos JM, Antonelli A, Bagheri HC (2013) Evolution of multicellularity coincided with increased diversification of cyanobacteria and the great oxidation event. Proc Natl Acad Sci. https://doi.org/10.1073/pnas.1209927110

Spang A, Stairs CW, Dombrowski N et al (2019) Proposal of the reverse flow model for the origin of the eukaryotic cell based on comparative analyses of Asgard archaeal metabolism. Nat Microbiol. https://doi.org/10.1038/s41564-019-0406-9

Stöver BC, Müller KF (2010) TreeGraph 2: combining and visualizing evidence from different phylogenetic analyses. BMC Bioinform. https://doi.org/10.1186/1471-2105-11-7

Yamamoto J, Shimizu K, Kanda T et al (2017) Loss of fourth electron-transferring tryptophan in animal (6-4) photolyase impairs DNA repair activity in bacterial cells. Biochemistry. https://doi. org/10.1021/acs.biochem.7b00366

Zhang M, Wang L, Zhong D (2017) Photolyase: dynamics and electron-transfer mechanisms of DNA repair. Arch Biochem Biophys. https://doi.org/10.1016/j.abb.2017.08.007 